\title{
Molecular chronicles of cytokine burst in patients with coronavirus disease 2019 (COVID-19) with cardiovascular diseases
}

Finosh G. Thankam, PhD, and Devendra K. Agrawal, PhD (Biochem), PhD (Med Sci), MBA

The emergence and pandemic spread of an acute respiratory disease caused due to a novel strain of SARS-Cov-2 (severe acute respiratory syndrome coronavirus 2), COVID-19 (coronavirus disease 2019) continues to threaten the global population. The increased rate of COVID-19 infection has resulted in a global health crisis and caused a huge economic burden in affected nations across the globe. The virus is believed to originate in bats and transmitted to humans possibly through an unknown carrier species in Wuhan, Hubei province, China, sometime in December 2019. ${ }^{1}$ However, proper scientific evidence is currently unavailable to prove/refute this theory, and extensive investigations have been initiated to unveil this mystery. Also, the entire world is hoping on medical sciences and medical research to tame COVID-19.

As of May 10, 2020, about 4.1 million confirmed cases with more than 282,500 deaths have been reported globally. The incidence in most affected countries as of May 10, 2020 , include 1.35 million cases and 80,246 deaths in the United States, $\sim 224,000$ cases and 26,478 deaths in Spain, $\sim 218,000$ cases and 30,395 deaths in Italy, $\sim 215,000$ cases and 31,587 deaths in the United Kingdom, and $~ 199,000$ cases and 1824 deaths in Russia. ${ }^{2}$ Unfortunately, the number of confirmed cases and mortality are increasing at an alarming rate. In addition, the recent studies revealed the basic reproduction number (R0) of COVID-19 to be around 2.2 , which is in the range of 1.4 to $6.5 .^{3}$ This suggests that each patient with COVID-19 could infect 2.2 people. Fundamentally, the $R_{O}$ values represent the spread of an infection and $R_{O}>1$ is an indication of population/community spread. ${ }^{4}$ The incubation period of COVID-19 after the transmission via inhalation or contact with infected droplets

\footnotetext{
From the Department of Translational Research, Western University of Health Sciences, Pomona, Calif

The research work of Dr Agrawal is supported by research grants R01HL128063, R01 HL144125, and R01HL147662 from the National Institutes of Health. The content of this article is solely the responsibility of the authors and does not necessarily represent the official views of the National Institutes of Health.

Received for publication April 26, 2020; revisions received May 12, 2020; accepted for publication May 16, 2020; available ahead of print June 6, 2020.

Address for reprints: Devendra K. Agrawal, PhD (Biochem), PhD (Med Sci), MBA, Department of Translational Research, Western University of Health Sciences, 309 E Second St, Pomona, CA 91766 (E-mail: DAgrawal@WesternU.edu).

J Thorac Cardiovasc Surg 2021;161:e217-26

$0022-5223 / \$ 36.00$

Copyright (c) 2020 by The American Association for Thoracic Surgery

https://doi.org/10.1016/j.jtcvs.2020.05.083
}

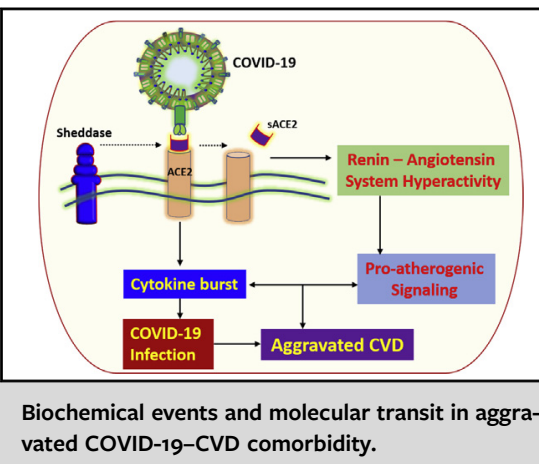

CENTRAL MESSAGE

The strong association between COVID-19 and CVD comorbidity points toward the COVID-19mediated dysregulation of ACE2 and subsequent activation of multiple proinflammatory signaling pathways.

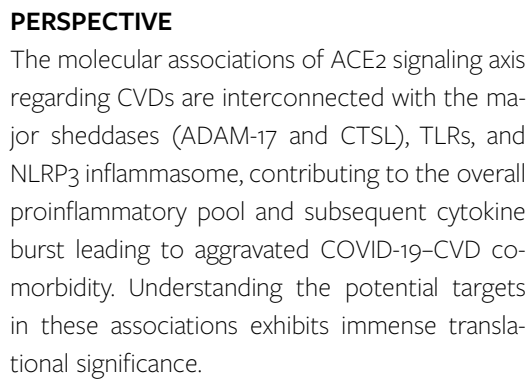

The molecular associations of ACE2 signaling axis regarding CVDs are interconnected with the major sheddases (ADAM-17 and CTSL), TLRS, and $\mathrm{NLRP}_{3}$ inflammasome, contributing to the overall proinflammatory pool and subsequent cytokine burst leading to aggravated COVID-19-CVD COmorbidity. Understanding the potential targets in these associations exhibits immense translational significance.

See Commentaries on pages e227 and e228. from the respiratory tract of infected individual is usually around 2 weeks, and the individual is asymptomatic during the initial incubation phase. ${ }^{1}$ Moreover, the usual symptoms include fever, dry cough, sore throat, fatigue, weakness, and breathing/swallowing difficulties. ${ }^{5}$ The disease is mild-to-moderate in healthy individuals; however, is severe-to-fatal in elderly population and those with other comorbidities, including diabetes and cardiovascular diseases (CVDs), leading to pneumonia, acute respiratory distress 
syndrome, and subsequently to organ failure. ${ }^{1}$ Supportive treatments are being administered to patients with COVID-19; however, specific antiviral agents are warranted.

On January 22, 2020, World Health Organization coined the term COVID-19 and Coronavirus Study Group of the International Committee defined COVID-19 as SARS-CoV-2 on February 11, 2020. Immediately after the outbreak in Wuhan, the Chinese researchers succeeded to isolate and sequence the COVID-19 genome, which opened further research opportunities in COVID-19 management. ${ }^{5}$ Apart from the respiratory system, COVID-19 has a severe pathologic impact on other organs, especially in the cardiovascular system, as evident from the increased risk of mortality and infection among patients with CVD. For instance, a recent case report from Washington State revealed congestive heart failure to be the second most common baseline comorbidity ( $\sim 43 \%$ ) next to the renal failure $(47.6 \%)$ in subjects with COVID-19. ${ }^{6}$ Specifically, cardiac arrhythmias, acute myocardial infarction (MI), myocarditis, and cardiac failure are the potential cardiovascular complications associated with COVID-19 infection. ${ }^{7-9}$ Unfortunately, very limited information is available on the underlying molecular mechanisms associated with the aggravated CVD pathology in patients with COVID-19. At this juncture, the major focus of this article is to provide insight into the possible molecular signaling, which may identify target(s) and pave multiple ways to intervene the CVD complications in subjects with COVID-19.

\section{ETIOLOGY AND TRANSMISSION}

COVID-19 belongs to coronaviruses, which are positive-stranded RNA viruses of the order Nidovirales, family Coronaviridae, and the sub-family Orthocoronavirinae. In general, the corona viruses are classified into 4 genera $(\alpha$-coronavirus, $\beta$-coronavirus, $\delta$-coronavirus, and $\gamma$-coronavirus). ${ }^{10}$ The genetic constitution studies and evolutionary analysis support bats and rodents to be the possible source of $\alpha$-coronavirus and $\beta$-coronavirus, whereas $\delta$-coronavirus and $\gamma$-coronavirus are expected to be derived from avian species. ${ }^{11}$ The characteristic crown-like morphology under the electron microscope due to the presence of spike glycoproteins on their surface covering the envelope reminiscent of the solar corona gave the name corona virus to this virus family because the word coronam means crown in Latin. ${ }^{11}$ COVID-19 is a $\beta$-coronavirus, which exists in round, elliptic, and pleomorphic morphology with average diameter of 60 to $140 \mathrm{~nm}$. Moreover, sensitivity to heat and ultraviolet rays are their characteristics. Hence, it is logical that COVID-19 can be effectively inactivated by organic solvents and detergents due to the solubility of constituent lipids from their envelope. ${ }^{11}$

COVID-19 shares $89 \%$ sequence identity with bat SARS-like-CoVZXC21 and $82 \%$ with human SARS-
CoV; therefore, it's named SARS-CoV-2. ${ }^{12}$ The phylogenic analysis based on receptor similarities revealed the possibilities of intermediate hosts, including pangolin, turtles, and snakes. ${ }^{13}$ The transmission of COVID-19 is human-tohuman, mainly among those who has intimate contact with the patients or incubation carriers; however, the transmission routine is still unknown. ${ }^{11}$ Symptomatic people have been the predominant source of COVID-19 spread, whereas the risk of transmission by pre-symptomatic/ asymptomatic patients is also very high. This suggests that the isolation of patients with COVID-19 and carriers is an ideal preventive measure to control the spread of COVID-19. In addition, aerosol transmission has been reported to be a potent means for COVID-19 transmission, which occurs via the respiratory aerosols discharged by patients while sneezing or coughing. ${ }^{5}$ The nasal droplets carry greater viral load and are reported to spread 1 to 2 meters in diameter and are deposited on surfaces. COVID-19 is capable of surviving on various surfaces for several days; however, it can be destroyed by the disinfectants within a minute. ${ }^{14}$ The possibilities of COVID-19 transmission via stool and contaminated water have also been hypothesized, which warrant further scientific proof. ${ }^{15}$ Even though the evidence for vertical transplantation is unavailable, a case of postnatal transmission has been reported. ${ }^{15}$ Moreover, it is believed that the proinflammatory microenvironment created by COVID-19 infection results in increased metabolic demand, leading to secondary complications including CVD malfunction. ${ }^{16}$ Hence, this could be the possible explanation for the increased mortality rate in elderly patients and patients who have inflammatory or metabolic disorders. Further studies are required to unveil the underlying molecular pathology.

In the current pandemic scenario of COVID-19, it is worthy to mention 2 major virulent strains; the $\mathrm{S}$ and $\mathrm{L}$ strains. $\mathrm{S}$ type is the ancestral strain from which $\mathrm{L}$ type was evolved due to the single-nucleotide polymorphism at position 8782. Alternatively, we are lacking proper scientific evidence whether the evolution of $\mathrm{L}$ strain occurred in humans or in the intermediate host. Furthermore, the L-type strain $(\sim 70 \%)$ exhibits more virulence and is more contagious when compared with the S strain $(\sim 30 \%))^{17}$ Also, the molecular mechanisms underlying the strain switch are unknown, which recommends further research.

\section{GENOME, STRUCTURE, AND PATHOPHYSIOLOGY}

COVID-19 is an enveloped positive-stranded RNA virus with a nucleocapsid. The +ssRNA of viral genome is $\sim 30$ $\mathrm{kb}$ long and contains a $5^{\prime}$-cap and $3^{\prime}$-poly-A tail, and the COVID-19 strain isolated from Wuhan is $29.9 \mathrm{~kb}^{18}$ COVID-19 ssRNA contains 29,891 nucleotides, with $38 \% \mathrm{G}+\mathrm{C}$ content encoding 9860 amino acids, and has 2 untranslated regions and open reading frames (ORFs). ${ }^{12}$ 
The genome contains 6 to 11 ORFs, where the two-thirds of viral genome is contained in the first ORF $(\mathrm{ORF} 1 \mathrm{a} / \mathrm{b})$, which codes for 2 polyproteins (ppla and pplab) and 16 non-structural proteins (Nsps). ${ }^{12,19}$ The functions and functional domains of the non-structural proteins are displayed in Figure 1, $A .{ }^{12}$ The structural and accessory proteins are coded by the remaining ORFs, including spike glycoprotein (SG), small envelope protein (SEP), nucleocapsid protein (NCP), and matrix protein (MP). ${ }^{20}$ The genome of COVID-19 is organized in the order of $5^{\prime}$-orf1/ab (replicase)-structural proteins (SG-SEP-MP-NCP)-3'. Interestingly, COVID-19 lacks the hemagglutinin-esterase gene that is characteristic of $\beta$-corona viruses. ${ }^{12}$ The frameshift between ORF1a and ORF1b drives the translation of ppla and pplab which, in turn, are proteolytically processed by viral-encoded chymotrypsin-like protease or main protease. In contrast, the $16 \mathrm{Nsps}$ are processed by papain-like proteases. ${ }^{11}$ The expression of COVID-19 proteins is facilitated by replication-transcription complex mediated through subgenomic RNAs. Also, the termination sequences are interspaced between ORFs. ${ }^{21}$ The genomic organization and the structural composition of COVID-19 are displayed in Figure $1, A$ and $B$.

Interestingly, the cumulative action of all structural proteins of COVID-19 and other coronaviruses are not required to assemble the infectious virion, suggesting their additional overlapping functions. ${ }^{22}$ For instance, most structural proteins are associated with various aspects of viral replication. SG mediates the attachment to the receptor and membrane fusion between COVID-19 and host cells. In addition, the SG binding facilitates viral infusion in the adjacent uninfected cells, suggesting that the activity of SG determines the transmission potential and tropism of COVID-19. ${ }^{23}$
Based on this observation, it is logical that the syncytia formation is the primary mechanism of direct viral entry between the host cells, destabilizing their neutralizing antibodies. ${ }^{24}$ The SG is composed of S1 and S2 domains. The $\mathrm{S} 1$ domain mediates receptor binding employing both $\mathrm{N}$ and $\mathrm{C}$ terminal ends and the $\mathrm{S} 2$ domain drives the fusion of COVID-19 with the host cell membrane. ${ }^{23}$ The 3-dimensional structural analysis of the $\mathrm{S} 1$ domain of COVID-19 revealed the presence of a core domain and an external subdomain. The external subdomain shares more similarities with SARS-COV unveiling angiotensinconverting enzyme 2 (ACE2) as the major receptor for COVID-19. ${ }^{23}$

Following membrane fusion using the S2 domain, the viral genome is released to the cytosol, where the uncoated viral genome initiates the translation of ppla and pplab. The pp1a and pp1ab code for Nsps required for the assembly of replication transcription complex in doublemembrane vesicle. ${ }^{25,26}$ Immediately following the assembly, the replication transcription complex initiates the replication of viral RNA to synthesize a battery of subgenomic RNAs encoding the structural and accessory proteins. Finally, employing the host endoplasmic reticulum (ER) and Golgi machinery, the newly formed viral genome and proteins assemble to form viral particle buds. Viral particle buds are virion-containing vesicles that ultimately fuse with the host cell plasma membrane to release the virus. ${ }^{27,28}$ COVID-19 shares similar molecular mechanisms with other $\beta$-coronaviruses, and the available information regarding COVID-19 infection cycle and pathology is mainly based on SARS-CoV. Hence, a detailed understanding of COVID-19 molecular pathology is warranted to develop effective management strategies.

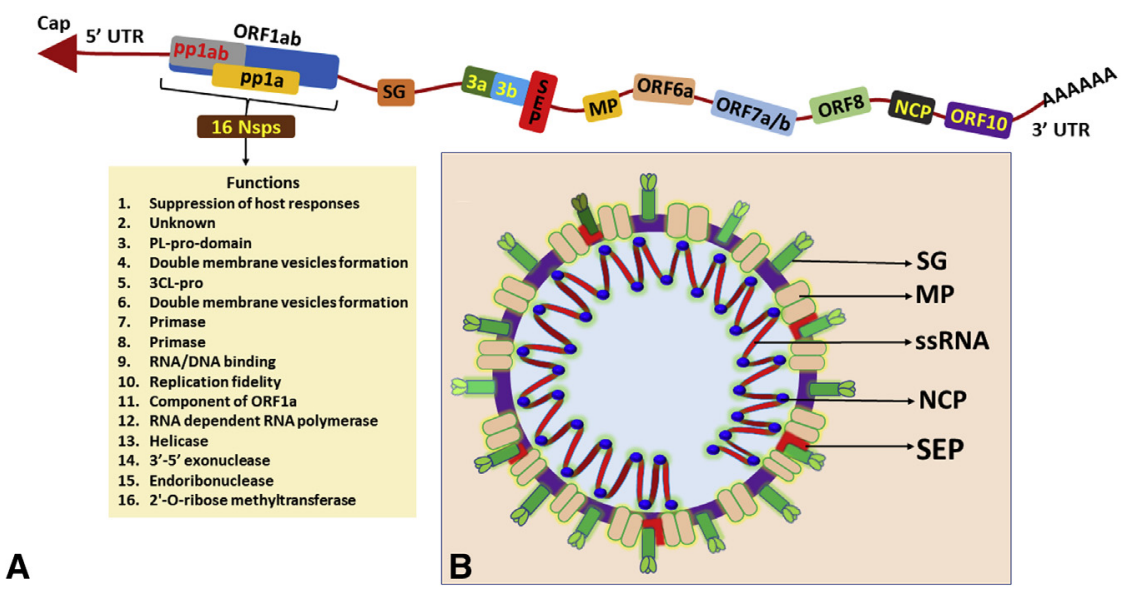

FIGURE 1. A, Genomic organization of COVID-19. The COVID-19 genome contains 6-11 ORFs where the two-thirds of viral genome is contained in the first ORF (ORF1a/b), which codes for 2 polyproteins (ppla and pplab) and 16 Nsps. The genome of COVID-19 is organized in the order of $5^{\prime}$-orf1/ab (replicase)-structural proteins (SG-SEP-MP-NCP)-3' . B, Structural organization of COVID-19: COVID-19 exists in round, elliptic, and pleomorphic morphology with average diameter of 60 to $140 \mathrm{~nm}$. The structural and accessory proteins including SG, SEP, NCP, and MP. ORF, Open reading frame; Nsp, nonstructural protein; $S G$, spike glycoprotein; $S E P$, small envelope protein; $M P$, matrix protein; $N C P$, nucleocapsid protein. 
The NCP is RNA genome binding protein that is primarily involved in the assembly and stability of nucleocapsid. In addition, NCP is associated with viral replication, host cell response, synthesis of virus-like particles, and virion formation. ${ }^{22}$ The shape of the viral envelope is defined by MP, which drives the virion assembly where the envelope formation is facilitated by the homotypic interactions of MP. ${ }^{29}$ In addition, MP interacts with SG to retain ER-Golgi intermediate compartment and/or Golgi complex for loading the viral particles. ${ }^{22}$ Furthermore, the interaction between MP and NCP exhibits multiple functions, including the stabilization of nucleocapsid and internal core of virions and the orchestration of viral assembly. ${ }^{22}$ Similarly, as the name implies, SEP is the smallest of the major structural proteins of coronaviruses. The drastic upregulation of SEP inside the host cell has been observed during the replication phase; however, limited amount is incorporated to the envelope. ${ }^{30}$ In addition, the SEP has been traced abundantly in ER, Golgi, and ER-Golgi intermediate compartment, suggesting its potential role in virion assembly and budding. ${ }^{31}$ In short, SEP and MP constitute the major part of the envelope, and their interactions play a significant role in the formation and release of virus-like particles. ${ }^{32}$ Moreover, recent studies on recombinant coronaviruses lacking SEP revealed poor propagation and minimal virulence, ${ }^{22}$ suggesting significant translational opportunities. A detailed understanding regarding the structural proteins and Nsps would open novel avenues in the development of potential therapeutic/ diagnostic targets to effectively manage COVID-19; however, further research on proteomics, genomics, and metabolomics is warranted to accomplish this goal. The biological events associated with COVID-19 infection are displayed in Figure 2, and further detailed information is described in the sections to follow.

\section{IMMUNOPATHOLOGY OF COVID-19}

Upon entry to the host cells, the coronaviruses are detected by the conventional pattern-recognition receptors as viral RNA acts as pathogen-associated molecular patterns. Generally, multiple intracellular Toll-like receptors (TLRs), including TLR3, TLR7, TLR8, and TLR9, detect viral genome in the endosomal vesicles. ${ }^{33}$ In addition, the viral genome in the cytosol is recognized by the cytosolic receptors, including retinoic-acid inducible gene I, melanoma differentiation-associated gene 5, and cyclic-GMPAMP synthase. ${ }^{5}$ The binding of viral ligands with the receptors initiates the recruitment and assembly of adaptor proteins, including TIR-domain-containing adaptor protein including interferon (IFN)- $\beta$, mitochondrial antiviralsignaling protein, and stimulator of IFN genes protein that trigger the activation of transcription factor nuclear factor $-\kappa \mathrm{B}(\mathrm{NF}-\kappa \mathrm{B})$ and IFN regulatory factor 3 via MyD88 adapter. ${ }^{34-36}$ Interferon regulatory factor 3 triggers the expression of IFN- $\alpha / \beta$, whereas NF- $\kappa \mathrm{B}$ stimulates the expression of a battery of proinflammatory cytokines that portray anti-viral defense by the host cells. ${ }^{36,37}$ However, dysregulation of innate immune response against virus results in immunopathology, as evident from the cytokine burst in patients with COVID-19. Evidently, the inflammatory mediators/cytokines/chemokines including interleukin (IL)-1, IL-2, IL-4, IL-6, IL-7, IL-10, IL-12, IL-13, IL-17, granulocyte-colony stimulating factor, macrophage colony-stimulating factor, IFN- $\gamma$-induced protein 10 , monocyte chemoattractant protein 1 , IFN- $\gamma$, tumor necrosis factor- $\alpha$ (TNF- $\alpha$ ), and others are drastically upregulated in patients with COVID-19. Also, the postmortem specimen from a patient with COVID-19 displayed extreme inflammatory events in the lung alveoli and exhibited severe lung injury. ${ }^{5}$ Together, COVID-19 infects the respiratory mucosa and epithelium followed by invasion to other cell types and subsequently elicits adverse immune responses leading to cytokine burst, which ultimately leads to a critical clinical condition or even death.

Like other $\beta$-coronaviruses, COVID-19 elicits a lethal cytokine burst, leading to human mortality. Even though inflammation is the first line of defense against viral infections, the hyperinflammation favors pro-viral features, including the spread of virus to other organs especially via the infiltrated immune cells and circulatory fluids. ${ }^{38}$ Among the highly upregulated proinflammatory cytokines with the predominance of IL- $1 \beta^{5}$ suggest the involvement of Nodlike receptor protein 3 (NLRP3) inflammasome activation and signaling in COVID-19 pathology. IL-1 $\beta$ is generated by the proteolytic cleavage of pro-IL- $1 \beta$ by caspase- 1 following the activation of NLRP3 inflammasome. NLRP3 assembly is triggered by discrete sensor proteins following the recognition of pathogen-associated molecular patterns /damage-associated molecular patterns by the cell surface receptors including TLR2, TLR4, and TREM1. The activation of NLRP3 recruits the adapter protein apoptosis-associated speck-like protein containing a caspase recruitment domain (ASC), which binds and activates caspase-1. Finally, the active NLRP3 inflammasome upregulates the transcription of pro-IL-1 $\beta$ gene and subsequent activation by caspase$1 .{ }^{39}$ Hence, it is reasonable to speculate that COVID-19 (and other coronaviruses) is capable of modulating inflammasome activation, as evident from significantly increased IL-1 $\beta$ expression. ${ }^{38}$ Evidently, the ion channel proteins SEP and ORF3a (also called $\mathrm{X} 1$ which is a $\mathrm{Na} / \mathrm{K}$ ion channel) from SARS-CoV activate NLRP3, suggesting a similar possible mechanism in COVID-19 pathology. The upregulation of ORF3a presents the key biochemical events associated with cytokine burst, including NF- $\kappa \mathrm{B}$ activation, Golgi fragmentation, ER stress, disturbed chemokine homeostasis, dysregulation of vesicle trafficking, and eventually cell death. Moreover, ORF3a interferes with IFN expression and signaling and upregulates fibrinogen, another inducer of cytokine burst. ${ }^{40}$ Interestingly, ORF3a requires 


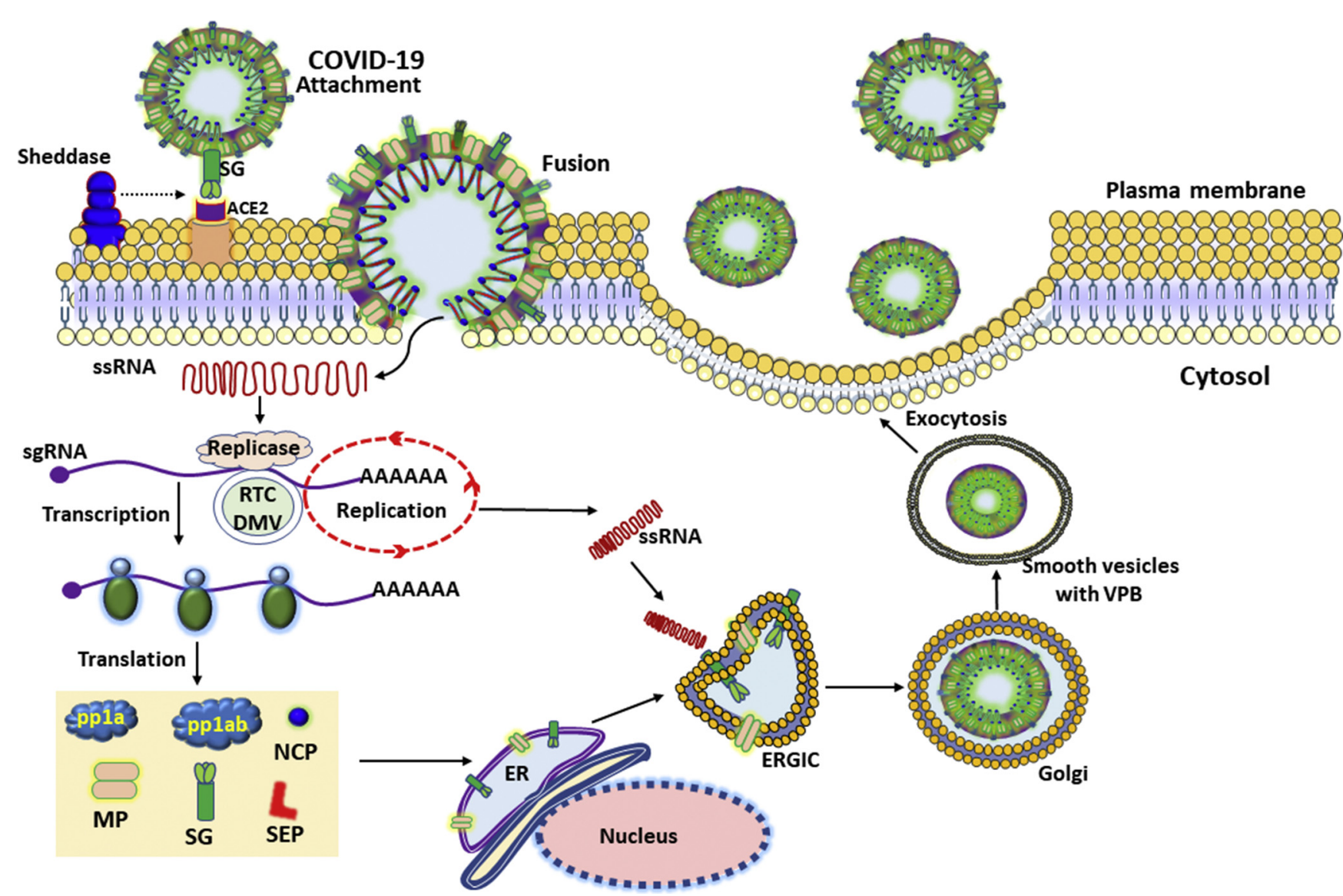

FIGURE 2. Pathology of COVID-19 infection. SG mediates the attachment of COVID-19 to its receptor, ACE2, in the plasma membrane of alveolar cells. The binding is facilitated by the sheddases (ADAM metallopeptidase domain-17/CTSL/transmembrane protease/serine subfamily member 2), leading to the membrane fusion between COVID-19 and host cells. The S1 subunit of SG is involved in the high-affinity binding of COVID-19 to the ACE2 receptor, whereas the S2 subunit facilitates the membrane fusion by harboring essential mediators. Following membrane fusion, the viral genome is released to the cytosol, where uncoated viral genome initiates the translation of ppla and pplab. The pp1a and pp1ab code for non-structural proteins required for the assembly of RTC in DMV. Immediately following the assembly, the RTC initiates the replication of viral RNA to synthesize a battery of sgRNAs encoding the structural and accessory proteins. Finally, employing the host ER and Golgi machinery, the newly formed viral genome and proteins assemble to form VPBs. VPBs are virion containing vesicles that ultimately fuse with the host cell plasma membrane to release virus. COVID-19, Coronavirus disease 2019; $S G$, spike glycoprotein; $A C E 2$, angiotensin-converting enzyme 2; sgRNA, subgenomic RNA; $R T C$, replication transcription complex; $D M V$, doublemembrane vesicle; $E R$, endoplasmic reticulum; $V P B$, viral particle buds; $M P$, matrix protein; $S G$, spike glycoprotein; $N C P$, nucleocapsid protein; $S E P$, small envelope protein; ERGIC, ER-Golgi intermediate compartment.

SEP for infection, which is evident from the nonviability of the recombinant coronavirus lacking ORF3a and SEP. ${ }^{41}$ Also, the NLRP3 signaling of ORF3a is independent of its ion channel function. ${ }^{40}$ Similar to SARS-CoV, ORF3a in COVID-19 is expected to trigger NLRP3 inflammasome by facilitating TNF receptor-associated factor 3-mediated ubiquitination of ASC and the downstream NF- $\kappa \mathrm{B}$ activation. ${ }^{40}$ However, detailed examination is warranted to understand the NLRP3 activation by COVID-19, which could open novel translational avenues.

\section{COVID-19 AND CVDs}

Patients with atherosclerotic heart disease may suffer ischemia in the context of COVID-19. Hence, the patients with chronic coronary artery disease/CVD are at greater risk of COVID-19 fatality due to increased susceptibility of developing acute coronary syndrome and MI. Specifically, COVID-19 infection results in increased energy/ metabolite demand, owing to increased cytokine burden by the surviving myocardium, which aggravates ischemic injury, leading to type II MI. Since the COVID-19 infection elicits severe "cytokine burst" resulting in the elevation of overall cytokine pool, this creates a drastic inflammatory burden in the cardiovascular system. The resultant acute inflammatory stress within the systemic vessels results in instability of atherosclerotic plaques and subsequent plaque rupture. Recent seminal studies revealed drastic increase in cardiac troponins ( $\mathrm{TnI}$ or $\mathrm{TnT}$ ) in the patients who 
developed myocardial injury following COVID-19 infection. Apart from the troponins, patients display increased levels of white blood cell count, C-reactive protein, and biomarkers of cardiac injury including creatine kinase, lactate dehydrogenase, myoglobin, and others. ${ }^{42,43}$ In short, recent reports consistently proclaim that the CVD comorbidities in COVID-19 markedly increase the adverse myocardial events and short-term mortality rate compared with naïve COVID-19 infection.

The upregulation of diverse proinflammatory cytokines including CRP, IL-6, IFN- $\gamma$, IL-1, and TNF- $\alpha$ suggests that cytokine burst is the major trigger in the damage of extrapulmonary organs/systems, including the cardiovascular system. ${ }^{44}$ ACE2, the receptor for COVID-19 SG, is a carboxypeptidase that plays a pivotal role in cardiovascular and immune system. Well known for its cardioprotective function, ACE2 is widely distributed in the heart and lung tissues, suggesting a possible association of the aggravated symptoms in patients with CVD. ${ }^{45}$ In addition, the impaired respiratory function and hypoxemia due to COVID-19 infection induce ischemic damage to myocardial cells. ${ }^{45}$ Based on these observations, it is logical to consider ACE2 as the bridge between the primary target organ (lungs) and the cardiovascular system. These findings suggest that ACE2 signaling acts as a possible transit of molecular pathways that interconnects cytokine burst and COVID-19-CVD comorbidity. Further investigation is required to establish the downstream signature pathways to unveil translational significance.

\section{ACE2 in CVDs}

The critical role of the renin-angiotensin system (RAS) in CVD has been well established and ACE2 is a negative regulator for RAS. ACE2 proteolytically inactivates angiotensin (Ang) I and Ang II and is predominantly expressed in the endothelium of heart and kidneys as well as several other cell types, including alveolar cells and vascular smooth muscle cells. ${ }^{46}$ ACE2 is a carboxypeptidase, located on the X chromosome, which acts on Ang I to form Ang 1-9 peptide, which is the precursor of the vasodilator Ang 1-7. Also, Ang 1-7 is the feedback inhibitor of Ang II. ${ }^{46,47}$ Being the major regulator of hypertension and a potent CVD risk factor, RAS functions in blood pressure homeostasis and electrolyte balance. ${ }^{48}$ As the increased activation of RAS is associated with CVDs, angiotensin-converting enzyme inhibitor (ACEi) and Ang II antagonists became effective therapeutics in the management of CVD pathology. However, based on current information, it is unclear whether patients with COVID-19 on ACEi and angiotensin receptor blockers (ARBs) do worst or better. However, the heptapeptide Ang 1-7 exerts beneficial effects on CVD, suggesting the contrasting roles of ACE2 and its isoform homologue ACE. Also, the ACE-2-Ang 1-7 axis prevents CVD pathology, suggesting that the alterations in the homeostasis between ACE-2-Ang 1-7 and ACE-Ang II axes determine the development and progression of CVDs. ${ }^{49}$

The binding of Ang II with type-1 receptor (AT1R) initiates a series of biochemical events leading to vasoconstriction, oxidative stress, fibrosis, and retention of electrolytes. In contrast, the ACE2-Ang 1-7 axis via G-protein coupled protein receptor, Mas, acts as a counter regulator of RAS by promoting vasodilation, antioxidant responses, and antifibrotic reactions. ${ }^{50}$ The decline in ACE2 expression following the progression of CVDs enhances myocardial dysfunction. In addition, decreased ACE2 activity triggers the activation of myocardial NADPH oxidase system, leading to superoxide-activated oxidative stress and hyperactivation of MMPs, which subsequently aggravate myocardial remodeling. ${ }^{51,52}$ Since ACE2 is responsible for counterbalancing the pathologic effects of Ang II and its metabolic clearance, the deficiency of ACE2 enhances Ang II and decreases Ang 1-7 in circulation and tissues. This leads to early-stage cardiac hypertrophy and fibrosis, which are reversible with ACEi and ARB treatment. ${ }^{53}$ Since ACE2 activation prevents the adverse post-MI cardiac events including left ventricular remodeling and fibrosis, the administration of ACE2 inhibitors are very risky, whereby the ACEi worsens CVD comorbidity in patients with COVID-19. However, ARBs may decrease the pathology induced by COVID-19 due to possible crossovers between ARBs and AT1R and AT2R. Also, ARBs interfere the production of chymase and Ang II. ${ }^{54}$ In addition, it is logical that the ACEi and ARB interfere with the binding of COVID-19 SG with the ACE2 receptors owing to their receptor affinity. However, the reports on the crossreactivity of ACEi and ARB with COVID-19 biomolecule components are unavailable and demand further attention. The association of COVID-19-CVD comorbidity based on ACE2 signaling is displayed in Figure 3.

\section{ACE2: THE "CELLULAR PORTAL" FOR COVID-19 ENTRY}

As discussed, the S1 subunit of SG is involved in the high-affinity binding of COVID-19 to ACE2 receptor, whereas the $\mathrm{S} 2$ subunit facilitates the membrane fusion by harboring the essential mediators. ${ }^{55,56}$ The binding induces conformational change in the SG which, in turn, increases its susceptibility to undergo proteolytic cleavage between $\mathrm{S} 1$ and $\mathrm{S} 2$ subunits. This suggests that the cleavage of SG by host proteases is essential for the entry of COVID-19 in the host cells. ${ }^{57}$ Interestingly, the cysteine protease cathepsin L (CTSL) has been identified to catalyze the cleavage of $\mathrm{SG}^{58}$ In addition, the membrane-bound ACE2 is cleaved by ADAM metallopeptidase domain-17 (ADAM-17, also called TNF- $\alpha$-converting enzyme, TNACE) to release a fully active soluble glycoprotein fraction. ${ }^{59}$ ADAM-17-induced ACE2 shedding results in the suppression of downstream signaling, leading to increased 


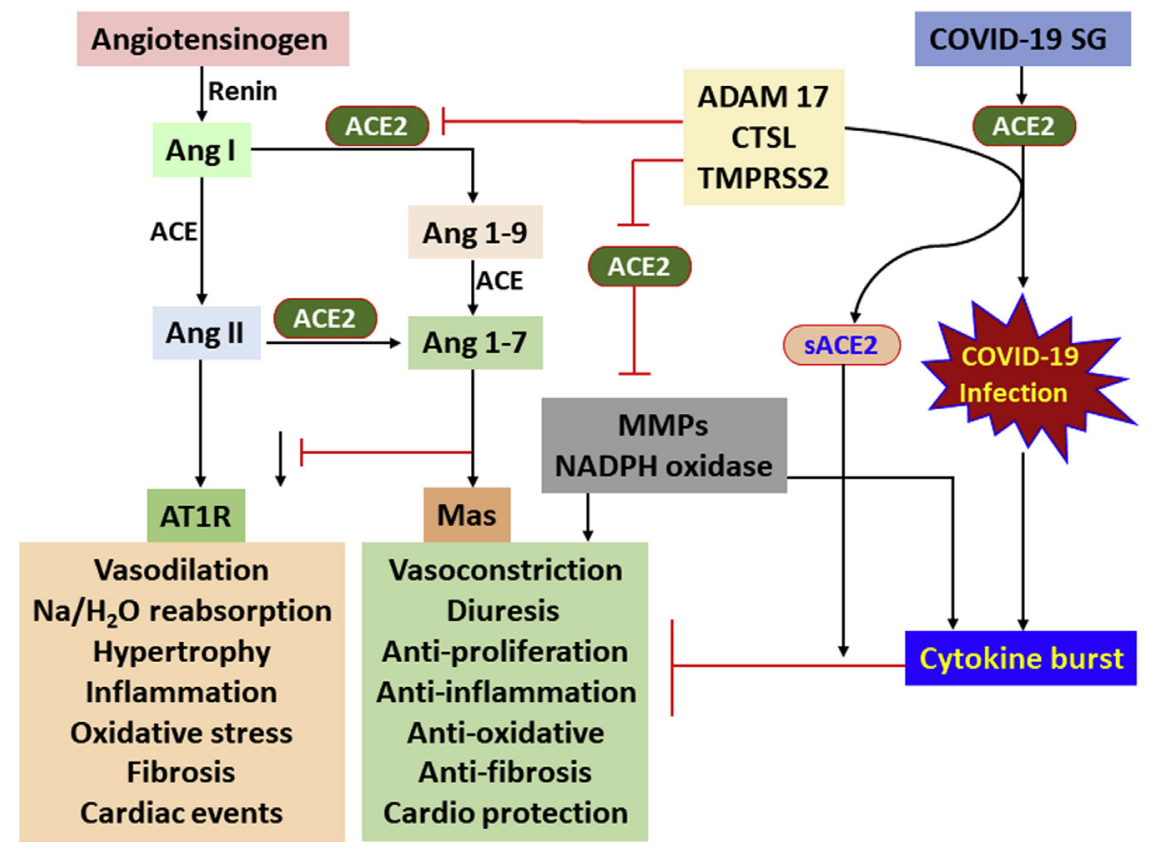

FIGURE 3. ACE2 signaling in CVD versus COVID-19 infection. ACE2 proteolytically inactivates Ang I and Ang II and acts on Ang I to form Ang 1-9 peptide, which is the precursor of the vasodilator Ang 1-7. The binding of Ang II with type-1 receptors (AT1R and AT2R) initiates a series of biochemical events leading to vasoconstriction, oxidative stress, fibrosis, and retention of electrolytes. In contrast, ACE2-Ang 1-7 axis via G-protein coupled protein receptor (Mas) acts as counter-regulator by promoting vasodilation, antioxidant responses, and antifibrotic reactions. The decline of ACE2 expression following the progression of CVDs enhances the myocardial dysfunction and induces inflammatory burden. The decreased ACE2 activity results in the activation of myocardial NADPH oxidase system, leading to superoxide-activated oxidative stress and hyperactivation of MMPs, which subsequently aggravate CVD pathology. The COVID-19 SG increases the susceptibility of SG to undergo proteolytic cleavage by sheddases, including ADAM-17, CTSL, and TMPRSS2, which is essential for coronavirus entry to the host cells. The shedding of ACE2 results in the suppression of downstream signaling, leading to increased Ang II level and subsequent aggravation of CVD pathology. sACE2 is associated with aggravated CVD pathology. Ang, Angiotensin; ACE2, angiotensin-converting enzyme 2; COVID-19, coronavirus disease 2019; SG, spike glycoprotein; ADAM-17, ADAM metallopeptidase domain-17; $C T S L$, cysteine protease cathepsin L; TMPRSS2, transmembrane protease/serine subfamily member 2; $M M P$, matrix metallopeptidase; $N A D P H$, nicotinamide adenine dinucleotide phosphate.

Ang II level and subsequent aggravation of CVD pathology. ${ }^{60}$ Alternatively, several other proteases especially the type II transmembrane serine proteases transmembrane protease/serine subfamily member 2 and human airway trypsin-like protease facilitate the cleavage of SG and promote the viral entry independent of CTSL and ADAM17. ${ }^{61,62}$ A similar mechanism is expected to occur in COVID-19 infection. ${ }^{63}$ Hence, it is reasonable to speculate that the engagement of ACE2 in COVID-19 fusion deviates from its cardioprotective function resulting in aggravated CVD-COVID-19 comorbidity (Figure 3). Future mechanistic studies would be helpful to derive a translationally significant conclusion.

\section{AGGRAVATED INFLAMMATION AND POSSIBLE MECHANISMS IN COVID-19-CVD COMORBIDITY}

The hyperactivation of RAS is tightly associated with the initiation and progression of CVDs whereas the activation of ACE2 exhibits immunomodulation since Ang 1-7 triggers anti-inflammatory and antioxidant responses. Possibly, Ang 1-7 elicits the immunomodulatory effects via the activation and downstream signaling of Mas and AT2R. In addition, the expression of ACE2 has been correlated with the proportionate reduction in the formation of atherosclerotic plaques. ${ }^{64}$ Interestingly, the SG protein and coronavirus infection significantly downregulate the expression/ activity of ACE2, which subsequently results in an enhanced local inflammatory milieu. In addition, downregulation of ACE2 builds up its secondary targets including bradykinin and vasoactive peptides, resulting in acute respiratory distress syndrome pathology. ${ }^{65}$ Based on these reports, it is logical that the increased use and shedding of ACE2 following COVID-19 infection results in ACE2 deficiency, which eventually paves the way for cytokine burst and CVD comorbidity via multiple pathways.

As the SG of COVID-19 negatively regulates ACE2 and facilitates the shedding of ACE2 ectodomain, the protective functions of ACE2 in the cardiovascular system of patients COVID-19-CVD with remain impaired. Hence, the downregulated ACE2 results in impaired RAS, resulting in enhanced vascular inflammation. ${ }^{66}$ In addition, the apoptotic cells following virus infection upregulates 


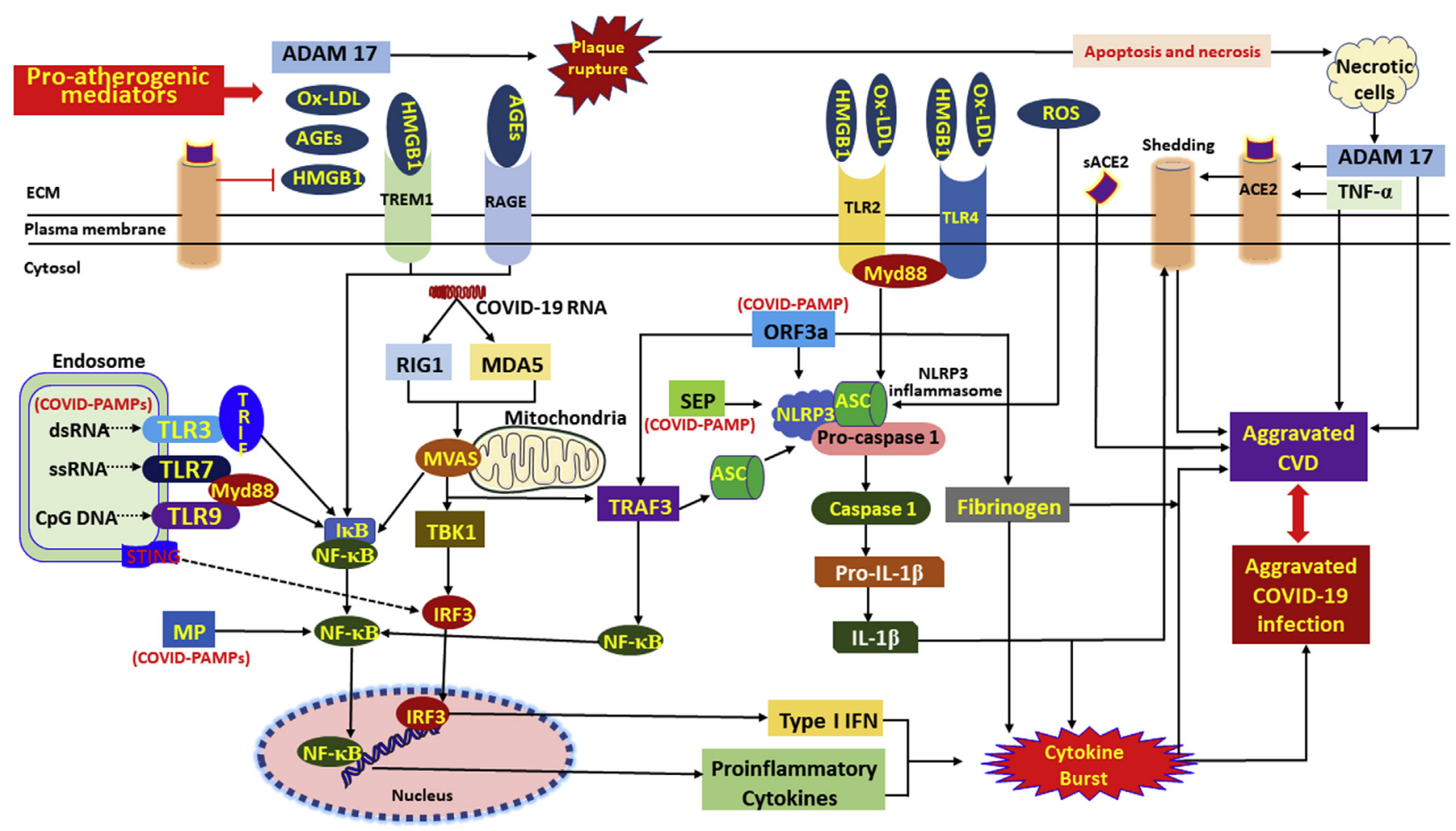

FIGURE 4. Proposed molecular mechanism underlying aggravated inflammatory response in COVID-19-CVD comorbidity. The components of COVID19, including the RNA and proteins, act as intracellular PAMPs, which are recognized by conventional pattern recognition receptors, especially TLRs, RIGI-like receptors (RLRs), and NLRP3 inflammasome. The TLRs, including TLR3, TLR7, TLR8, and TLR9, detect viral genome in the endosomal vesicles. In addition, the viral genome in the cytosol are recognized by the cytosolic receptors including RIG-1 and MDA5. The binding of viral ligands with the receptors initiates the recruitment and assembly of adaptor proteins including TRIF, MAVS, and STING, which trigger the activation of the transcription factor $\mathrm{NF}-\kappa \mathrm{B}$ and IRF3 via MyD88 adapter. IRF3 triggers the expression of type I IFNs, whereas NF- $\kappa$ B stimulates the expression of a battery of proinflammatory cytokines leading to cytokine burst. IL- $1 \beta$ is generated by the proteolytic activation of pro-IL- $1 \beta$ by caspase- 1 following the activation of NLRP3 inflammasome. The active NLRP3 inflammasome upregulates the transcription of pro-IL- $1 \beta$ gene and subsequent activation by caspase- 1 . The COVID-19 proteins including MP, SEP, and ORF3a activate NLRP3 via TRAF3 and subsequent IL-1 $\beta$ and ORF3a activate NF- $\kappa$ B and downstream cytokine burst. The apoptotic/necrotic cells following virus infection upregulate ADAM-17, the major sheddase for ACE2. IL-1 $\beta$ and TNF- $\alpha$ enhance the ACE2 shedding. The resultant SACE2 is a potent mediator for vascular inflammation and CVD pathology. ADAM-17 activates atherosclerotic plaque rupture and vascular inflammation. ACE2 inhibits the DAMPs, including HMGB1 released from the infected and ischemic/necrotic cells due to membrane damage. The decreased levels of ACE2 lead to increased DAMPs, especially OxLDL, HMGB1, AGEs, and ROS. These mediators trigger NLRP3 inflammasome via TLR2, TLR4, RAGE, and/or TREM1 axes in cardiovascular system. The upregulation of such DAMPs in the ACE2-depleted environment is detrimental, resulting in aggravated COVID-19-CVD comorbidity. ADAM-17, ADAM metallopeptidase domain-17; OxLDL, oxidized low-density lipoprotein; AGEs, advanced glycation end products; $H M G B 1$, high mobility group box 1; TLR, Toll-like receptor; ROS, reactive oxygen species; $A C E 2$, angiotensin-converting enzyme 2; TNF- $\alpha$, tumor necrosis factor- $\alpha$; COVID-19, coronavirus disease 2019; TRIF, TIR-domain-containing adaptor protein including IFN- $\beta$; RIG-1, retinoic acid-inducible gene 1; MDA5, melanoma differentiation-associated gene 5; STING, stimulator of interferon genes protein; $M P$, matrix protein; $M A V$, mitochondrial antiviral-signaling protein; TRAF3, TNF receptor-associated factor; $P A M P s$, pathogen-associated molecular patterns; $N F-\kappa B$, nuclear factor- $\kappa \mathrm{B}$; $I R F 3$, interferon regulatory factor 3; ORF, open reading frame; $S E P$, small envelope protein; $N L R P 3$, Nod-like receptor protein 3; IL, interleukin; IFN, interferon; $C V D$, cardiovascular disease.

ADAM-17, the major sheddase for ACE2.$^{67}$ Moreover, the product of NLRP3 activation, IL- $1 \beta$, and the potent proinflammatory mediator TNF- $\alpha$ were reported to enhance ACE2 shedding. ${ }^{66}$ Furthermore, ADAM-17 has been adversely associated with atherosclerotic plaque rupture and vascular inflammation, resulting in poor clinical outcomes. ${ }^{68,69}$ In addition, the proinflammatory environment associated with hyperlipidemia in CVDs favors the upregulation of ADAM-17, which reveals the increased susceptibility of cardiovascular events in aged populations. ${ }^{70}$ Also, the pathologic role of NLRP3 inflammasome in CVD, ischemic/nonischemic heart diseases, and MI has been well established. ${ }^{71,72}$ Interestingly, ACE2 inhibits damage-associated molecular patterns including HMGB1 released from infected and ischemic/necrotic cells due to membrane damage. Being a potent trigger for NLRP3 inflammasome via TLR2, TLR4, RAGE, and/or TREM1 axes in cardiovascular system, the upregulation of 
HMGB1 in the ACE2 depleted environment is detrimental, resulting in aggravated COVID-19-CVD comorbidity. ${ }^{73,74}$ Taken together, the cumulative proinflammatory events elicited by the COVID-19 infection and the proinflammatory status of the host tissues as well as the age-related factors aggravate CVD comorbidity, which is evident by the increased mortality rate.

The mounting evidence from COVID-19 as well as related coronavirus infections revealed the possibilities of multiple biological events pointing toward the association of aggravated COVID-19-CVD comorbidity. The key events derived from the extensive literature survey suggest that: (1) the hyperinflammatory microenvironment resulting from COVID-19 infection and CVD pathology aggravates the cytokine burst; (2) the ACE2 dysregulation resulting from COVID-19 SG and the host sheddases especially CTSL and ADAM-17 are markedly greater in patients with CVD which aggravate the comorbidity; (3) intracellular TLR signaling builds up a cellular proinflammatory milieu, which accelerates COVID-19 propagation and cytokine burst; (4) multiple proinflammatory events due to COVID-19 infection and CVD immunopathology converge to NF- $\kappa$ B activation and subsequent expression of a battery of proinflammatory genes contributing to cytokine burst; and (5) activation of NLRP3 inflammasome by SEP and ORF3a represents another mode of COVID-19-mediated cytokine burst, and NLRP3 activation due to oxidative stress, oxidized-LDL and other triggers in CVD patients synergistically enhance proinflammatory status, leading to aggravated COVID-19-CVD comorbidity. The proposed molecular signaling underlying the aggravated COVID19-CVD comorbidity is portrayed in Figure 4.

\section{CONCLUSIONS}

Herein, we present the possible molecular associations by focusing on the ACE2 signaling axis regarding CVDs by interconnecting the major sheddases (ADAM-17 and CTSL), TLRs, and NLRP3 inflammasome as the major transit point contributing to the overall proinflammatory pool and subsequent cytokine burst leading to aggravated COVID-19-CVD comorbidity. However, in vitro, in vivo, in silico, and clinical assessments are warranted on these aspects to derive novel translational avenues in the effective management of COVID-19-CVD comorbidity. Also, the identification of novel targets to address the severity of symptoms in COVID-19 infection in patients in CVD is needed urgently. As COVID-19 continues to threaten humanity, the entire world is hoping on medical research outcomes with effective strategies to tame this pandemic spread. To conclude, an interdisciplinary approach by merging the knowledge and efforts of a myriad of health science researchers is warranted to effectively address the health concerns raised by the pandemic COVID-19.

\section{Conflict of Interest Statement}

The authors reported no conflicts of interest.

The Journal policy requires editors and reviewers to disclose conflicts of interest and to decline handling or reviewing manuscripts for which they may have a conflict of interest. The editors and reviewers of this article have no conflicts of interest.

\section{References}

1. Singhal T. A review of coronavirus disease-2019 (COVID-19). Indian J Pediatr. 2020;87:281-6.

2. COVID-19. Coronavirus (COVID-19) map. Available at: https://google.com/ covid19-map. Accessed May 10, 2020.

3. Liu Y, Gayle AA, Wilder-Smith A, Rocklöv J. The reproductive number of COVID-19 is higher compared to SARS coronavirus. J Travel Med. 2020;27: taaa021.

4. Fraser C, Donnelly CA, Cauchemez S, Hanage WP, Van Kerkhove MD, Hollingsworth TD, et al. Pandemic potential of a strain of influenza A (H1N1) early findings. Science. 2009;324:1557-61.

5. Guo Y-R, Cao Q-D, Hong Z-S, Tan YY, Chen SD, Jin HJ, et al. The origin, transmission and clinical therapies on coronavirus disease 2019 (COVID-19) outbreak - an update on the status. Mil Med Res. 2020;7:11.

6. Arentz M, Yim E, Klaff L, Sharukh L, Francis XR, Maria C, et al. Characteristics and outcomes of 21 critically ill patients with COVID-19 in Washington State. JAMA. 2020;323:1612-4

7. Zhang J-J, Dong X, Cao Y-Y, Yuan Y, Yang Y, Yan Y-Q, et al. Clinical characteristics of 140 patients infected with SARS-CoV-2 in Wuhan, China. Allergy. February 19, 2020 [Epub ahead of print].

8. Wang D, Hu B, Hu C, Zhu F, Liu X, Zhang J, et al. Clinical characteristics of 138 hospitalized patients with 2019 novel coronavirus-infected pneumonia in Wuhan, China. JAMA. 2020;323:1061-9.

9. Xu X-W, Wu X-X, Jiang X-G, Xu K-J, Ying L-J, Ma C-L, et al. Clinical findings in a group of patients infected with the 2019 novel coronavirus (SARS-Cov-2) outside of Wuhan, China: retrospective case series. BMJ. 2020; 368:m606.

10. Chan JF-W, To KK-W, Tse H, Jin D-Y, Yuen K-Y. Interspecies transmission and emergence of novel viruses: lessons from bats and birds. Trends Microbiol. 2013; 21:544-55.

11. Cascella M, Rajnik M, Cuomo A, Dulebohn SC, Di Napoli R. Features, evaluation and treatment coronavirus (COVID-19). In: StatPearls. StatPearls Publishing; 2020. Available at: http://www.ncbi.nlm.nih.gov/books/NBK554776/. Accessed March 31, 2020.

12. Chan JF-W, Kok K-H, Zhu Z, Chu H, To KK, Yuan S, et al. Genomic characterization of the 2019 novel human-pathogenic coronavirus isolated from a patien with atypical pneumonia after visiting Wuhan. Emerg Microbes Infect. 2020;9: 221-36.

13. Liu Z, Xiao X, Wei X, Li J, Yang J, Tan H, et al. Composition and divergence of coronavirus spike proteins and host ACE2 receptors predict potential intermediate hosts of SARS-CoV-2. J Med Virol. 2020;92:595-601.

14. Kampf G, Todt D, Pfaender S, Steinmann E. Persistence of coronaviruses on inanimate surfaces and their inactivation with biocidal agents. J Hosp Infect. 2020;104:246-51.

15. Chen H, Guo J, Wang C, Luo J, Yu X, Zhang W, et al. Clinical characteristics and intrauterine vertical transmission potential of COVID-19 infection in nine pregnant women: a retrospective review of medical records. Lancet Lond Engl. 2020; 395:809-15.

16. Rali AS, Sauer AJ. COVID-19 Pandemic and cardiovascular disease. US Cardiol Rev. 2020;14:e01.

17. Tang X, Wu C, Li X, Song Y, Yao X, Wu X, et al. On the origin and continuing evolution of SARS-CoV-2. Natl Sci Rev. 2020:nwaa036.

18. Wu F, Zhao S, Yu B, Chen Y-M, Wang W, Song Z-G, et al. A new coronavirus associated with human respiratory disease in China. Nature. 2020;579:265-9.

19. Song Z, Xu Y, Bao L, Zhang L, Yu P, Qu Y, et al. From SARS to MERS, thrusting coronaviruses into the spotlight. Viruses. 2019;11:59.

20. Cui J, Li F, Shi Z-L. Origin and evolution of pathogenic coronaviruses. Nat Rev Microbiol. 2019;17:181-92.

21. Perlman S, Netland J. Coronaviruses post-SARS: update on replication and pathogenesis. Nat Rev Microbiol. 2009;7:439-50. 
22. Schoeman D, Fielding BC. Coronavirus envelope protein: current knowledge. Virol J. 2019;16.

23. Lu R, Zhao X, Li J, Niu P, Yang B, Wu H, et al. Genomic characterisation and epidemiology of 2019 novel coronavirus: implications for virus origins and receptor binding. Lancet. 2020;395:565-74.

24. Fehr AR, Perlman S. Coronaviruses: an overview of their replication and pathogenesis. Methods Mol Biol Clifton NJ. 2015;1282:1-23.

25. de Wilde AH, Snijder EJ, Kikkert M, van Hemert MJ. Host Factors in coronavirus replication. Curr Top Microbiol Immunol. 2018;419:1-42.

26. Sawicki SG, Sawicki DL. Coronavirus transcription: a perspective. Curr Top Microbiol Immunol. 2005;287:31-55.

27. Hussain S, Pan J, Chen Y, Yang Y, Xu J, Peng Y, et al. Identification of novel subgenomic RNAs and noncanonical transcription initiation signals of severe acute respiratory syndrome coronavirus. J Virol. 2005;79:5288-95.

28. Perrier A, Bonnin A, Desmarets L, Danneels A, Goffard A, Rouillé Y, et al. The C-terminal domain of the MERS coronavirus M protein contains a trans-Golgi network localization signal. J Biol Chem. 2019;294:14406-21.

29. Masters PS. The molecular biology of coronaviruses. Adv Virus Res. 2006;66; 193-292.

30. Venkatagopalan P, Daskalova SM, Lopez LA, Dolezal KA, Hogue BG. Coronavirus envelope (E) protein remains at the site of assembly. Virology. 2015;478: 75-85.

31. Nieto-Torres JL, Dediego ML, Alvarez E, Jiménez-Guardeño JM, Regla-Nava JA, Llorente M, et al. Subcellular location and topology of severe acute respiratory syndrome coronavirus envelope protein. Virology. 2011;415:69-82.

32. Baudoux P, Carrat C, Besnardeau L, Charley B, Laude H. Coronavirus pseudoparticles formed with recombinant $\mathrm{M}$ and $\mathrm{E}$ proteins induce alpha interferon synthesis by leukocytes. J Virol. 1998;72:8636-43.

33. Wu J, Chen ZJ. Innate immune sensing and signaling of cytosolic nucleic acids Annu Rev Immunol. 2014;32:461-88.

34. Seth RB, Sun L, Ea C-K, Chen ZJ. Identification and characterization of MAVS, a mitochondrial antiviral signaling protein that activates NF- $\kappa \mathrm{B}$ and IRF3. Cell. 2005; 122:669-82.

35. Ishikawa H, Barber GN. STING is an endoplasmic reticulum adaptor that facilitates innate immune signalling. Nature. 2008;455:674-8.

36. Kawai T, Akira S. The role of pattern-recognition receptors in innate immunity: update on Toll-like receptors. Nat Immunol. 2010;11:373-84.

37. Takeuchi O, Akira S. Innate immunity to virus infection. Immunol Rev. 2009;227: 75-86.

38. Gram AM, Frenkel J, Ressing ME. Inflammasomes and viruses: cellular defence versus viral offence. J Gen Virol. 2012;93:2063-75.

39. Thankam FG, Roesch ZK, Dilisio MF, Radwan MM, Kovilam A, Gross RM, et al. Association of inflammatory responses and ECM disorganization with HMGB1 upregulation and NLRP3 inflammasome activation in the injured rotator cuff tendon. Sci Rep. 2018;8:1-14.

40. Siu K-L, Yuen K-S, Castaño-Rodriguez C, Ye Z-W, Yeung M-L, Fung S-Y, et al. Severe acute respiratory syndrome coronavirus ORF3a protein activates the NLRP3 inflammasome by promoting TRAF3-dependent ubiquitination of ASC. FASEB J. 2019:33:8865-77.

41. Castaño-Rodriguez C, Honrubia JM, Gutiérrez-Álvarez J, DeDiego ML, NietoTorres JL, Jimenez-Guardeño JM, et al. Role of severe acute respiratory syndrome coronavirus viroporins $\mathrm{E}, 3 \mathrm{a}$, and $8 \mathrm{a}$ in replication and pathogenesis. mBio. 2018:9.

42. Guo T, Fan Y, Chen M, Wu X, Zhang L, He T, et al. Cardiovascular implications of fatal outcomes of patients with coronavirus disease 2019 (COVID-19). JAMA Cardiol. March 27, 2020 [Epub ahead of print].

43. Shi S, Qin M, Shen B, Cai Y, Liu T, Yang F, et al. Association of cardiac injury with mortality in hospitalized patients with COVID-19 in Wuhan, China. JAMA Cardiol. March 25, 2020 [Epub ahead of print].

44. Huang C, Wang Y, Li X, Ren L, Zhao J, Hu Y, et al. Clinical features of patients infected with 2019 novel coronavirus in Wuhan, China. Lancet. 2020;395: 497-506.

45. Zheng Y-Y, Ma Y-T, Zhang J-Y, Xie X. COVID-19 and the cardiovascular system. Nat Rev Cardiol. 2020;17:259-60.

46. Oudit GY, Crackower MA, Backx PH, Penninger JM. The role of ACE2 in cardiovascular physiology. Trends Cardiovasc Med. 2003;13:93-101.

47. Vickers C, Hales P, Kaushik V, Gavin J, Tang J, Godbout K, et al. Hydrolysis of biological peptides by human angiotensin-converting enzyme-related carboxypeptidase. J Biol Chem. 2002;277:14838-43.

48. Zaman MA, Oparil S, Calhoun DA. Drugs targeting the renin-angiotensinaldosterone system. Nat Rev Drug Discov. 2002;1:621-36.
49. Raizada MK, Ferreira AJ. ACE2: a new target for cardiovascular disease therapeutics. J Cardiovasc Pharmacol. 2007;50:112-9.

50. Wang W, Bodiga S, Das SK, Lo J, Patel V, Oudit GY. Role of ACE2 in diastolic and systolic heart failure. Heart Fail Rev. 2012;17:683-91.

51. Tikellis C, Bernardi S, Burns WC. Angiotensin-converting enzyme 2 is a key modulator of the renin-angiotensin system in cardiovascular and renal disease. Curr Opin Nephrol Hypertens. 2011;20:62-8.

52. Bodiga S, Zhong JC, Wang W, Basu R, Lo J, Liu GC, et al. Enhanced susceptibility to biomechanical stress in ACE2 null mice is prevented by loss of the p47phox NADPH oxidase subunit. Cardiovasc Res. 2011;91:151-61.

53. Chamsi-Pasha MAR, Shao Z, Tang WHW. Angiotensin-converting enzyme 2 as a therapeutic target for heart failure. Curr Heart Fail Rep. 2014;11:58-63.

54. Rolf JD. Clinical characteristics of Covid-19 in China. N Engl J Med. 2020;382: 1860.

55. Gallagher TM, Buchmeier MJ. Coronavirus spike proteins in viral entry and pathogenesis. Virology. 2001;279:371-4.

56. Hofmann H, Pöhlmann S. Cellular entry of the SARS coronavirus. Trends Microbiol. 2004;12:466-72.

57. Li F, Berardi M, Li W, Farzan M, Dormitzer PR, Harrison SC. Conformational states of the severe acute respiratory syndrome coronavirus spike protein ectodomain. J Virol. 2006;80:6794-800.

58. Simmons G, Gosalia DN, Rennekamp AJ, Reeves JD, Diamond SL, Bates P. Inhibitors of cathepsin $\mathrm{L}$ prevent severe acute respiratory syndrome coronavirus entry. Proc Natl Acad Sci USA. 2005;102:11876-81.

59. Burrell LM, Harrap SB, Velkoska E, Patel SK. The ACE2 gene: its potential as a functional candidate for cardiovascular disease. Clin Sci. 2013;124:65-76.

60. Mukerjee S, Gao H, Xu J, Sato R, Zsombok A, Lazartigues E. ACE2 and ADAM17 interaction regulates the activity of presympathetic neurons. Hypertension. 2019;74:1181-91.

61. Matsuyama S, Nagata N, Shirato K, Kawase M, Takeda M, Taguchi F. Efficient activation of the severe acute respiratory syndrome coronavirus spike protein by the transmembrane protease TMPRSS2. J Virol. 2010;84:12658-64.

62. Shulla A, Heald-Sargent T, Subramanya G, Zhao J, Perlman S, Gallagher T. A transmembrane serine protease is linked to the severe acute respiratory syndrome coronavirus receptor and activates virus entry. J Virol. 2011;85:873-82.

63. Guo J, Huang Z, Lin L, Lv J. Coronavirus Disease 2019 (COVID-19) and cardiovascular disease: a viewpoint on the potential influence of angiotensin-converting enzyme inhibitors/angiotensin receptor blockers on onset and severity of severe acute respiratory syndrome coronavirus 2 infection. J Am Heart Assoc. 2020;9:e016219.

64. Tikellis C, Thomas MC. Angiotensin-converting enzyme 2 (ACE2) is a key modulator of the renin angiotensin system in health and disease. Int J Pept. 2012;2012:256294

65. Jia HP, Look DC, Tan P, Shi L, Hickey M, Gakhar L, et al. Ectodomain shedding of angiotensin converting enzyme 2 in human airway epithelia. Am J Physiol Lung Cell Mol Physiol. 2009;297:L84-96.

66. Fu Y, Cheng Y, Wu Y. Understanding SARS-CoV-2-mediated inflammatory responses: from mechanisms to potential therapeutic tools. Virol Sin. March 3, 2020 [Epub ahead of print].

67. Scheller J, Chalaris A, Garbers C, Rose-John S. ADAM17: a molecular switch to control inflammation and tissue regeneration. Trends Immunol. 2011;32:380-7.

68. Xu J, Mukerjee S, Silva-Alves CRA, Carvalho-Galvão A, Cruz JC, Balarini CM, et al. A disintegrin and metalloprotease 17 in the cardiovascular and central nervous systems. Front Physiol. 2016;7:469.

69. Chemaly M, McGilligan V, Gibson M, Clauss M, Watterson S, Alexander HD, et al. Role of tumour necrosis factor alpha converting enzyme (TACE/ ADAM17) and associated proteins in coronary artery disease and cardiac events. Arch Cardiovasc Dis. 2017;110:700-11

70. Liu H, Wang H, Cheng D, Wang Q, Pei Z, Zhu N, et al. Potential role of a disintegrin and metalloproteinase-17 (ADAM17) in age-associated ventricular remodeling of rats. RSC Adv. 2019;9:14321-30.

71. Zhou W, Chen C, Chen Z, Liu L, Jiang J, Wu Z, et al. NLRP3: a novel mediator in cardiovascular disease. J Immunol Res. 2018;2018:5702103.

72. An N, Gao Y, Si Z, Zhang H, Wang L, Tian C, et al. Regulatory mechanisms of the NLRP3 Inflammasome, a novel immune-inflammatory marker in cardiovascular diseases. Front Immunol. 2019;10:1592.

73. Qi YF, Zhang J, Wang L, Zhang H, Wang L, Tian C, et al. Angiotensin-converting enzyme 2 inhibits high-mobility group box 1 and attenuates cardiac dysfunction post-myocardial ischemia. J Mol Med Berl Ger. 2016;94:37-49.

74. Thankam FG, Dilisio MF, Dietz NE, Agrawal DK. TREM-1, HMGB1 and RAGE in the shoulder tendon: dual mechanisms for inflammation based on the coincidence of glenohumeral arthritis. PLoS One. 2016;11:e0165492. 